\title{
Application of a CdTe Detector for Measurements of Mammographic X-ray Spectra
}

\author{
$\mathrm{J} \mathrm{C} \mathrm{SANTOS}^{{ }^{1 *}, \mathrm{~A} \mathrm{TOMAL}^{2} \text { AND P R COSTA }}{ }^{1}$. \\ ${ }^{1}$ Instituto de Física, Universidade de São Paulo, SP, Brazil \\ ${ }^{2}$ Instituto de Física Gleb Wataghin, Universidade Estadual de Campinas, SP, Brazil \\ *Email: josilene@usp.br
}

Published online: August 07, 2017

The Author(s) 2017. This article is published with open access at www.chitkara.edu. in/publications

\begin{abstract}
This work aims to characterize mammographic x-ray beams incident and transmitted by breast phantoms (from 0 to $45 \mathrm{~mm}$ ) composed from known proportion of glandular and adipose tissue-equivalent materials. This study was performed for mammographic $\mathrm{x}$-ray beams generated by a mammography equipment using different target/filter combinations ( $\mathrm{Mo} / \mathrm{Mo}, \mathrm{Mo} / \mathrm{Rh}$ and $\mathrm{W} / \mathrm{Rh}$ ). It was studied the modification of spectra shape of the beams transmitted through different thicknesses of these materials. It was also evaluated the penetrability of these transmitted beams by its correlations to the HVL, which were experimentally estimated and derived from the $\mathrm{x}$-ray spectra measured using a spectrometry system with a CdTe detector. The x-ray spectra transmitted by the phantom with higher density presented lower intensity than those transmitted by those with lower density, as expected. The differences between the HVL values derived from the spectra and those estimated using air kerma measurements are lesser than $6 \%$ for about $88 \%$ of the spectra measured in this work. The expected spectra variations with phantom thickness, revealed by the measured transmitted x-ray spectra, were also confirmed by HVL measurements and agree with the estimated attenuation curves. The motivation of the study was related to the robustness of the spectra as a descriptor of radiation beams and the possibility of using these transmitted spectra for dose assessment related to mammographic procedures. We can conclude that developed method is able to characterize mammographic x-ray beams making it possible the use of this kind of data for dose assessment in mammography.
\end{abstract}

Keywords: CdTe detector; Mammography; x-ray spectra measurements

\section{INTRODUCTION}

The x-ray spectra quantify the amount of photons and its energies in a x-ray beam. It allows the calculation of derived quantities, generally applied for

Journal of Nuclear

Physics, Material Sciences, Radiation and

Applications

Vol-5 No-1

August 2017 pp. 91-102 
Santos, J.C. Tomal, A. Costa, P.R. quality control and patient dose assessment, such as half value layer (HVL), air kerma, mean energy and others (Meyer et al. (2004)). An accurate representation of $\mathrm{x}$-ray spectra used for radiological purposes is important for modeling the x-ray imaging process (Stumbo et al. (2004)). It allows the conversion of exposure information in dosimetric quantities for dose/image quality optimization (Bernhardt et al. (2006)). In the case of mammography, other applications of $\mathrm{x}$-ray spectra have been reported in literature such as, radiation shielding calculation and evaluation of x-ray tube voltage (Künzel et al. (2004); Künzel et al. (2008)). Evaluation of mammographic radiation beam qualities and image properties have also been previously presented byTomal et al. $(2013,2014)$.

The knowledge of primary and transmitted x-ray spectra by materials simulators of breast tissues is very important once it can provide information about the composition and attenuation properties of these materials (Byng et al. (1998); Geraldelli et al. (2013); Johns and Yaffe (1987)). This information is essential for dose estimation. Moreover, these spectra can also provide information to characterize the source emitter of $\mathrm{x}$-ray beams.

Measurement of clinical mammography $\mathrm{x}$-ray spectra present practical limitations mainly due to the high photon fluence produced by mammography equipments. Therefore, a common method to represent these spectra is using analytical or semi-empirical approaches (Blough et al. (1998); Boone et al. (1997); Hernandez and Boone (2014); Tucker et al. (1991)). However, this kind of spectrum becomes possible to be experimentally available after the advent of portable spectrometry system, which is capable to measure relatively high photon fluence. Mammographic x-ray spectra measured using these instruments generally requires complex alignment procedures, the use of pinholes and electrical or mechanical adjustments on the mammographic equipment. Different approaches have been reported in the literature (Matsumoto et al., 2000; Miyajima and Imagawa, 2002; Stumbo et al., 2004; Abbene et al., 2012; Zhang et al., 2012). Santos et al. (2017) have first introduced a study about the experimental x-ray spectra measurements in mammographic clinical equipments without changes in the original settings of these devices. Based on the Santos et al. (2017) methods the present work describes measurement of $\mathrm{x}$-ray spectra to characterize mammographic $\mathrm{x}$-ray beam transmitted by two different equivalent-tissue breast phantoms. The motivation of the study was related to the robustness of the spectra as a descriptor of radiation beams and the possibility of using these transmitted spectra for dose assessment related to mammographic procedures. Additionally, the possibility of measuring these spectra in clinical environment is relevant since it should represent realistically the radiation beams used in clinical practice. 
This study was performed using mammographic x-ray beams generated using different target/filter combinations ( $\mathrm{Mo} / \mathrm{Mo}, \mathrm{Mo} / \mathrm{Rh}$ and $\mathrm{W} / \mathrm{Rh}$ ) incident on the breast phantoms composed by a known proportion of glandular and adipose tissue-equivalent materials. The $\mathrm{x}$-ray spectra were measured using different thickness of the phantoms allowing the correlation of its morphology variation with the attenuator material. The results of this study can serve as a basis of future methods for dosimetry in mammography.

\section{MATERIAL AND METHODS}

Measurements of $\mathrm{x}$-ray spectra and attenuation curves were performed in order to characterize the transmitted mammographic beams by different thicknesses of two tissue-equivalent breast phantoms. In this study, it was used $\mathrm{x}$-ray beams generated using $28 \mathrm{kVp}$ and each of the target/filter combinations $(\mathrm{Mo} / \mathrm{Mo}, \mathrm{Mo} / \mathrm{Rh}$ and $\mathrm{W} / \mathrm{Rh}$ ) available in a Mammomat 3000 Nova (Siemens AG, Munich, DEU) mammographic equipment. Breast phantoms with two different tissue-equivalent compositions (CIRS Inc, model 012A) were positioned between the primary $\mathrm{x}$-ray beam and the spectrometer, without the compression paddle, for measurements of transmitted spectra. These phantom compositions are equivalent to a breast 30/70 (30\% of glandular and $70 \%$ of adipose tissue) and a 50/50 (50\% of glandular and 50\% of adipose tissue). In this work, the phantom equivalent of a 30/70 breast is referred as CIRS30/70 and the phantom equivalent of a 50/50 is referred as CIRS50/50. For each tissue-equivalent composition, the slabs of the materials were superimposed intercepting the primary beam in order to assemble thicknesses between 5 $\mathrm{mm}$ to $45 \mathrm{~mm}$. Aluminum transmission curves were also measured for each target/filter and tissue-equivalent composition combinations. The flowchart presented in Figure 1 summarizes the tube parameters and experimental setup used for data acquisition.

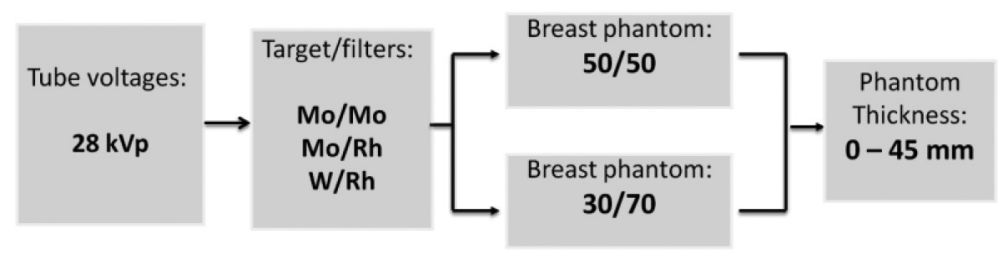

Figure 1: Summary of the tube parameters and experimental setup used for the production of transmitted $\mathrm{x}$-ray beam for which $\mathrm{x}$-ray spectra and attenuation curves were acquired.
Application of a CdTe Detector for Measurements of Mammographic X-ray Spectra 
Santos, J.C.

Tomal, A.

Costa, P.R.

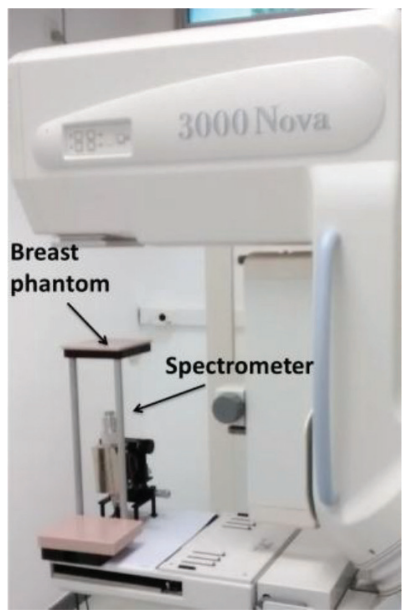

Figure 2: Spectrometer system and breast phantom positioned upon the breast support in a Mammomat 3000 Nova equipment, for transmitted x-ray spectra measurements.

The transmitted x-ray spectra were measured using a spectrometer model XR-100T (Amptek, Inc., Bedford, MA) with a $9 \mathrm{~mm}^{2}$ CdTe detector. The data processing was done by means of a digital pulse processor model PX4, also manufactured by Amptek. The detector was coupled to $25 \mu \mathrm{m}$ to $100 \mu \mathrm{m}$ collimator diameters, in order to reduce the detected photon flux and the scattered photon contribution on the detected beam. The collimator aperture was chosen depending on the thickness of the attenuation material. Figure 2 shows the spectrometer system positioned upon the breast support in a Mammomat 3000 Nova equipment, for transmitted x-ray spectra measurement. At this position, the CdTe crystal was at $20.5 \mathrm{~cm}$ from the breast support. The lower base of the breast phantom was positioned above the spectrometer in a plastic support, such as a frame, at $32 \mathrm{~cm}$ from the breast support. For this mammography equipment, the source to the positioning assembly is fixed at $65 \mathrm{~cm}$.

The energy calibration of the spectrometer was done using standard radiation sources of ${ }^{241} \mathrm{Am},{ }^{152} \mathrm{Eu}$ and ${ }^{133} \mathrm{Ba}$. The measured transmitted $\mathrm{x}$-ray spectra were corrected by the detector efficiency, estimated using Monte Carlo method (Tomal et al. (2015)), Compton distortion and fluorescent escape fraction were reduced or removed using stripping procedure (Di Castro et al. (1984); Santos et al. (2016)). In this way, the corrected x-ray spectra are presented in units of fluence normalized by the current-time product (photons/ $\mathrm{mm}^{2}$.mAs.keV).

For each x-ray spectrum, corresponding air kerma measurements were performed using a dedicated mammography ionization chamber model 10x56M (Radcal Corp., Monrovia, USA). This same detector was used to measure 


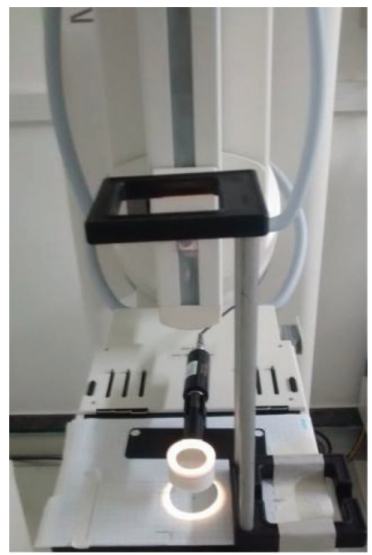

(a)

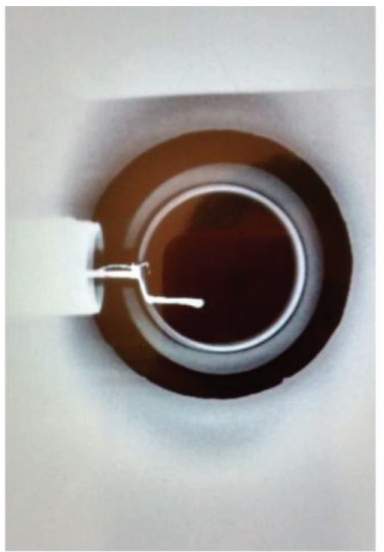

(b)

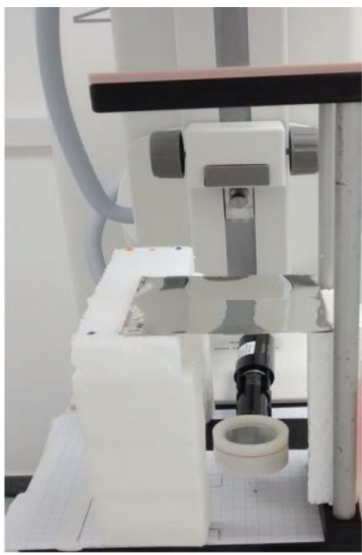

(c)

Figure 3: Experimental setup for air kerma measurements for the derivation of attenuation curves and HVL values highlighting the chamber positioning in the light field (a) and in the x-ray field (b) of the mammography equipment. (c), presents the positioning of al foils and the breast phantom.

transmission curves by aluminum. For these measurements, the $\mathrm{x}$-ray field was collimated using a lead plate with a hole of approximately $1.6 \mathrm{~cm}$ positioned next to the tube collimators. Figure 3 presents the ionization chamber positioned for the air kerma measurements used to derive the attenuation curves and HVL. Figure 3 (a) presents the ionization chamber positioned at 4.5 $\mathrm{cm}$ above the breast support in the light field of the mammography equipment delimited by the lead plate used. Figure 3 (b) presents the radiographic image of this detector positioned over the breast support such as shown in Figure 3 (a). Figure 3 (c) presents the positioning of the aluminum filters and the breast phantom for air kerma measurements.

The experimental data for the beam attenuation by aluminum were fitted using Archer's model (Archer et al. (1983)) in order to estimate the transmission curves. The HVL values corresponding to each $\mathrm{x}$-ray spectra measured were derived from these transmission curves. HVL values were also derived from experimental spectra and compared to those experimentally obtained using ionization chamber in order to assess the feasibility of obtaining such quantity from x-ray spectra. For this calculation, first the air kerma spectra were computed from the photon fluence spectra, $N(E)$, as showed in Equation (1).

$$
N_{k}(E)=N(E) \times\left(\frac{\mu_{t r}(E)}{\rho}\right)_{\text {air }} \times E
$$


Santos, J.C. Tomal, A. Costa, P.R.

Where is the photon fluence spectrum as a function of the energy, $E$, and are the mass energy transfer coefficients for air. Second, the air kerma transmitted by different thicknesses, $x$, of aluminum was computed analytically. The sum of these distributions is equivalent to the value of air kerma, of a x-ray beam transmitted for different thicknesses of aluminum:

$$
K_{a r}(x)=\sum N_{k}(E) e^{-\left(\frac{\mu(E)}{\rho}\right)_{A l} \cdot \rho_{A l} \cdot x} \cdot \Delta E
$$

Where are the mass attenuation coefficients for aluminum. Finally, the ratio was computed for each $x$ value used. Then, the HVL values were obtained using cubic spline interpolation of values in order to find the $x$ value (HVL) which satisfies the following relation:

$$
\frac{K_{a r}(x=H V L)}{K_{a r}(0)}=0.5
$$

The variation of $x$-ray spectra and its respective HVL values with the phantom thickness was analyzed for each anode/filter combination in order to characterize the typical beams transmitted for material that represents different thicknesses of breast tissue.

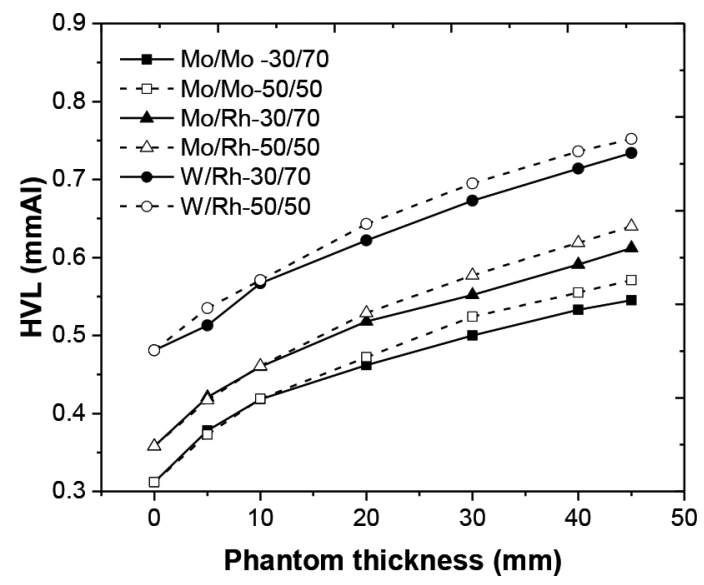

Figure 4: HVL values obtained for transmitted $\mathrm{x}$-ray spectra measured for each $\mathrm{Mo} / \mathrm{Mo}$, $\mathrm{Mo} / \mathrm{Rh}$ and $\mathrm{W} / \mathrm{Rh}$ target/filter combination as a function of the phantoms thicknesses. The filled and unfilled symbols represents the HVL values obtained for the $\mathrm{x}$-ray spectra transmitted for the breast phantoms Cirs30/70 and Cirs50/50, respectively. These values represent the experimental HVL, estimated from measurements using ionization chamber. The maximum uncertainty obtained for these values was $5 \%$. 


\section{RESULTS AND DISCUSSIONS}

The HVL values obtained from transmitted x-ray spectra measured for $\mathrm{Mo} /$ $\mathrm{Mo}, \mathrm{Mo} / \mathrm{Rh}$ and $\mathrm{W} / \mathrm{Rh}$ target/filter combination are illustrated in Figure 4. These results are presented as function of the phantoms thicknesses. The filled and unfilled symbols represents the HVL values obtained for the $\mathrm{x}$-ray spectra transmitted for the breast phantoms CIRS30/70 and CIRS50/50, respectively. It can be noted that, for the same target/filter combination, the use of the phantom with higher glandular tissue-equivalent content intercepting the radiation beam results in transmitted beams with higher HVL values and consequently higher effective energy relative to those transmitted by the material with lower glandular tissue-equivalent content. It can also be observed that the transmitted W/Rh beams are the most penetrating and Mo/Mo beams are the less penetrating. The knowledge of the power of penetration of these beams is very important for consideration of energy dependence in dosimeters used for depth dose calculations.

Figure 5 presents the transmission curves that represent the normalized air kerma for $\mathrm{x}$-ray spectra transmitted through 5 to $45 \mathrm{~mm}$ of tissue equivalent phantoms CIRS30/70 and CIRS50/50 relative to the air kerma produced by the incident photons on the surface of these materials. These results were obtained for incident beams generated using $28 \mathrm{kVp}$ different anode/filters combinations. This figure shows that the air kerma reduction occurs with increasing phantom thickness and this reduction is more pronounced for phantom with larger proportion of glandular tissue (CIRS50/50). The W/Rh radiation beams proved to be harder than $\mathrm{Mo} / \mathrm{Mo}$ and $\mathrm{Mo} / \mathrm{Rh}$ beams. These

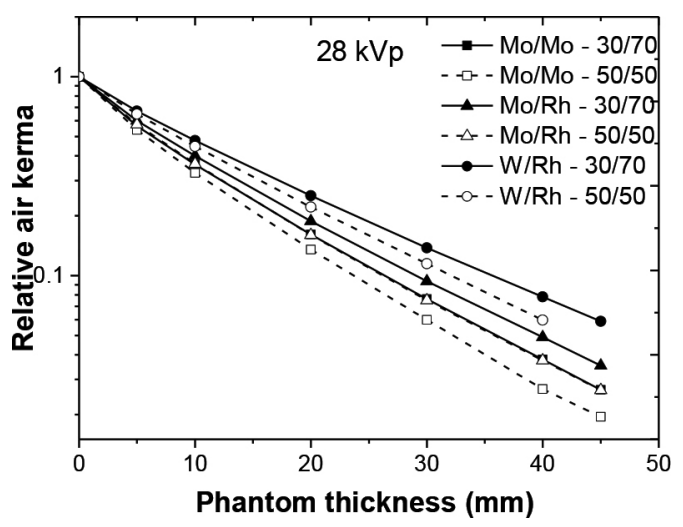

Figure 5: Relative air kerma transmission by 0 to $45 \mathrm{~mm}$ of tissue equivalent phantoms Cirs30/70 and Cirs50/50. It was used incident beams generated using $28 \mathrm{kVp}$ and Mo/ $\mathrm{Mo}, \mathrm{Mo} / \mathrm{Rh}$ and $\mathrm{W} / \mathrm{Rh}$ anode/filter combinations.
Application of a

CdTe Detector for

Measurements of

Mammographic

X-ray Spectra

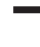


Santos, J.C.

Tomal, A.

Costa, P.R.

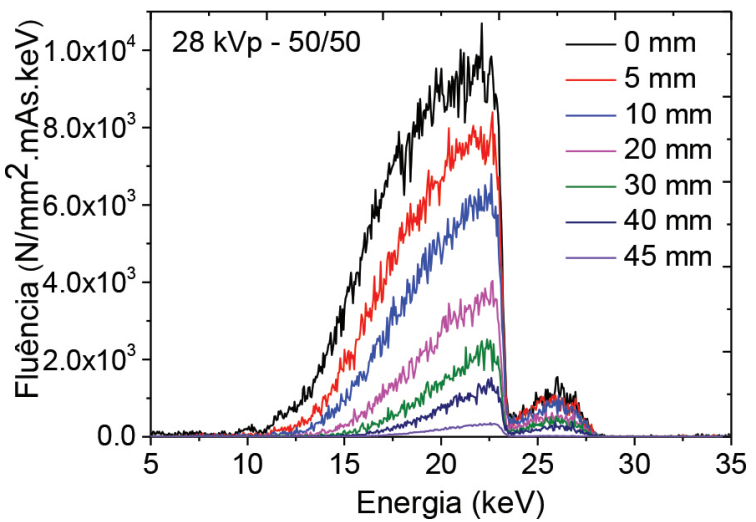

Figure 6: Transmitted photon fluence (photons $/ \mathrm{mm}^{2} . \mathrm{mAs} . \mathrm{keV}$ ) by 0 to $45 \mathrm{~mm}$ of tissue-equivalent phantom Cirs50/50. The x-ray incident beam was generated using $28 \mathrm{kVp}$ and $\mathrm{W} / \mathrm{Rh}$ target/filter combination.

results are in accordance with those found with measured transmitted x-ray spectra presented in Figure 4.

Figure 6 presents the photon fluence spectra transmitted by 0 to $45 \mathrm{~mm}$ of tissue-equivalent phantom CIRS50/50 for W/Rh combination at $28 \mathrm{kVp}$. The spectra intensities presented in this figure decrease with increasing of material thickness. In this way, the spectrum with higher intensity corresponds to the primary beam, and that with the lower intensity corresponds to the beam transmitted by $45 \mathrm{~mm}$ of the attenuator material. Similar results were obtained for $\mathrm{Mo} / \mathrm{Mo}$ and $\mathrm{Mo} / \mathrm{Rh}$ target/filter combination for both breast phantoms used (CIRS50/50 and CIRS30/70).

Figure 7 presents the comparisons between fluence spectra transmitted by the same thickness of the breast phantoms used in this study. These comparisons refer to the transmitted spectra by $20 \mathrm{~mm}$ of the breast phantoms (Figure 7 (a)), $30 \mathrm{~mm}$ (Figure 7 (b)), $40 \mathrm{~mm}$ (Figure 7 (c)) and $45 \mathrm{~mm}$ (Figure 7 (d)). The spectra transmitted by the material with composition equivalent to a breast 30/70 were plotted using black lines and those transmitted by the material equivalent to a breast 50/50 were represented using red lines lines.

Table 1 presents the HVL values (mmAl) calculated from air kerma measurements performed using ionization chamber (Experimental HVL) and those derived from the measured x-ray spectra. This quantity was calculated for all spectra measured in this study considering different target/filter combination and the several thicknesses of the breast phantoms used. The HVL values derived from the spectra presented a difference to those estimated using air kerma measurements less than $6 \%$ for about $88 \%$ of the spectra used in this work. 
Table 1: HVL values (mmAl) calculated from air kerma measurements performed using ionization chamber (Experimental HVL) and those derived from the measured X-ray spectra.

\begin{tabular}{|c|c|c|c|c|c|c|}
\hline \multicolumn{7}{|c|}{ CIRS30/70 } \\
\hline & \multicolumn{3}{|c|}{ Experimental HVL (mmAl) } & \multicolumn{3}{|c|}{$\begin{array}{l}\text { HVL Derived from spectra } \\
\qquad(\mathrm{mmAl})\end{array}$} \\
\hline $\begin{array}{c}\text { Phantom } \\
\text { thickness } \\
(\mathrm{mm})\end{array}$ & Mo/Mo & $\mathrm{Mo} / \mathrm{Rh}$ & $\mathrm{W} / \mathrm{Rh}$ & Mo/Mo & $\mathrm{Mo} / \mathrm{Rh}$ & $\mathrm{W} / \mathrm{Rh}$ \\
\hline 0 & $0.312(4)$ & $0.358(2)$ & $0.481(9)$ & $0.326(2)$ & $0.371(2)$ & $0.498(2)$ \\
\hline 5 & $0.378(1)$ & $0.421(1)$ & $0.535(1)$ & $0.391(3)$ & $0.433(3)$ & $0.546(3)$ \\
\hline 10 & $0.418(1)$ & $0.460(9)$ & $0.567(3)$ & $0.421(2)$ & $0.462(3)$ & $0.578(3)$ \\
\hline 20 & $0.462(2)$ & $0.518(20)$ & $0.622(1)$ & $0.470(4)$ & $0.522(4)$ & $0.664(3)$ \\
\hline 30 & $0.500(4)$ & $0.552(2)$ & $0.673(4)$ & $0.501(4)$ & $0.568(4)$ & $0.674(4)$ \\
\hline 40 & $0.533(2)$ & $0.591(9)$ & $0.714(7)$ & $0.538(3)$ & $0.607(4)$ & $0.716(5)$ \\
\hline 45 & $0.545(1)$ & $0.612(6)$ & $0.734(7)$ & $0.549(5)$ & $0.621(4)$ & $0.754(4)$ \\
\hline \multicolumn{7}{|c|}{ CIRS50/50 } \\
\hline 0 & $0.312(4)$ & $0.358(2)$ & $0.481(23)$ & $0.326(2)$ & $0.371(2)$ & $0.498(2)$ \\
\hline 5 & $0.373(3)$ & $0.417(2)$ & $0.513(4)$ & $0.393(3)$ & $0.436(3)$ & $0.542(3)$ \\
\hline 10 & $0.419(7)$ & $0.461(2)$ & $0.571(1)$ & $0.425(3)$ & $0.470(3)$ & $0.570(4)$ \\
\hline 20 & $0.472(7)$ & $0.529(1)$ & $0.643(1)$ & $0.474(5)$ & $0.517(5)$ & $0.629(5)$ \\
\hline 30 & $0.524(17)$ & $0.577(5)$ & $0.695(30)$ & $0.495(8)$ & $0.539(7)$ & $0.658(9)$ \\
\hline 40 & $0.555(25)$ & $0.619(4)$ & $0.736(2)$ & $0.501(11)$ & $0.536(10)$ & $0.669(11)$ \\
\hline 45 & $0.571(3)$ & $0.640(2)$ & $0.752(8)$ & $0.567(5)$ & $0.643(4)$ & $0.731(5)$ \\
\hline
\end{tabular}

Once the glandular tissue is denser than the adipose tissue (Hammerstein et al. (1979)) the spectra transmitted by the phantom with composition 50/50 has lower intensity than those transmitted by the phantom with composition $70 / 30$, as expected. It can be noticed that the difference between the intensities of these spectra for the materials attenuator used increases with increasing phantom thickness. It occurs because of the ratio of the number of photons transmitted by the phantom CIRS30/70 to those transmitted by the phantom CIRS50/50 for a given energy, should increase exponentially as a first approximation, in complying with the exponential attenuation law for monoenergetic beams.
Application of a CdTe Detector for Measurements of Mammographic X-ray Spectra 
Santos, J.C.
Tomal, A.
Costa, P.R.

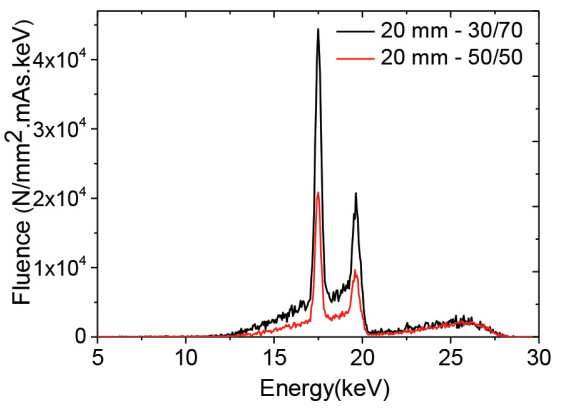

(a)

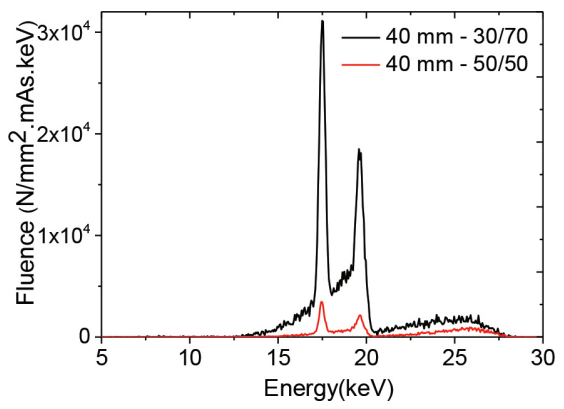

(c)

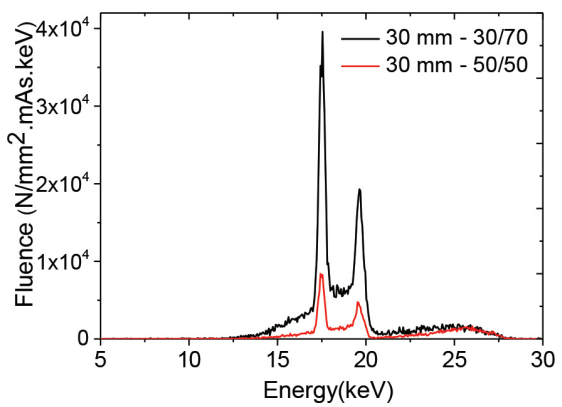

(b)

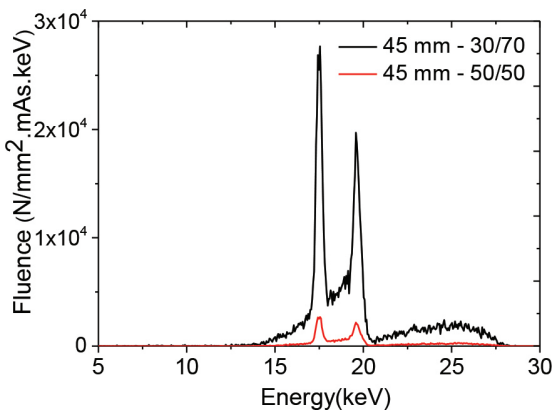

(d)

Figure 7: Comparisons between $x$-ray spectra transmitted by some thicknesses of two different breast phantoms: (a) $20 \mathrm{~mm}$, (b) $30 \mathrm{~mm}$, (c) $40 \mathrm{~mm}$ and (d) $45 \mathrm{~mm}$.

\section{CONCLUSIONS}

We tested a portable spectrometry system with CdTe detector to measure the $\mathrm{x}$-ray spectra from radiation beams generated by a mammography equipment and transmitted by breast phantoms in order to characterize these spectra that represents the distribution of the unattenuated photons when breast of different thicknesses and composition were irradiated for examination.

HVL measurement is a common method for characterization of $x$-ray beams for quality control and for assessment of equipment performance. In this study we have introduced a more sophisticated method to do this characterization, since from the $\mathrm{x}$-ray spectra, many quantities of dosimetric interest, such as dose, HVL, mean energy, etc, can be derived. It is important to notice that the modifications expected to the transmitted beam for different target/filter combination and different attenuator material extracted from the measured transmitted x-ray spectra were consistently confirmed by HVL measurements and agreed with the estimated transmission curves. Therefore, it can be 
concluded that the developed method was able to adequately characterize mammographic x-ray beams.

Once a method for transmitted spectra measurements is established, depth dose variation in these materials can be evaluated. Furthermore, these $\mathrm{x}$-ray spectra can be used to derive absorbed dose and mean glandular dose at various depth of these phantoms. The knowledge of HVL and the effective energy transmitted by different tissue compositions and combinations of various target/filter also allow the development and characterization of appropriate detectors which facilitates more efficient production of clinical images.

\section{ACKNOWLEDGEMENTS}

The authors thank the CAPES and FAPESP funding agencies for the grants received during the realization of this project. They thank FAPESP for funding this work [grant numbers 2013/07117-0; 2010/12237-7 and 2015/21873-8], the CNPq for support project [grant numbers 483170/2013-5; 312029/2009-8 and 309745/2015-2] and CNPQ/FAPESP for funding of the project INCT Metrology of Ionizing Radiation in Medicine [Grant number 2008/57863-2]. They also thank CDTN/CNEN for allowing the use of Mammography Laboratory to perform the experiments and Dr. Kwan Hoong $\mathrm{Ng}$ for his important comments and assistance on the production of this manuscript.

\section{REFERENCES}

[1] Archer, B.R., Thornby, J.I., Bushong, S.C., 1983. Diagnostic X-ray shielding design based on an empirical model of photon attenuation. Health Phys 44, 507-517.

[2] Bernhardt, P., Mertelmeier, T., Hoheisel, M., 2006. X-ray spectrum optimization of full-field digital mammography: Simulation and phantom study. Medical Physics 33, 4337-4349.

[3] Blough, M.M., Waggener, R.G., Payne, W.H., Terry, J.A., 1998. Calculated mammographic spectra confirmed with attenuation curves for molybdenum, rhodium, and tungsten targets. Med. Phys. 25, 1605-1612.

[4] Boone, J.M., Fewell, T.R., Jennings, R.J., 1997. Molybdenum, rhodium, and tungsten anode spectral models using interpolating polynomials with application to mammography. Med. Phys. 24, 1863-1874.

[5] Byng, J.W., Mainprize, J.G., Yaffe, M.J., 1998. X-ray characterization of breast phantom materials. Physics in Medicine and Biology 43, 1367.

[6] Di Castro, E., Pani, R., Pellegrini, R., Bacci, C., 1984. The use of cadmium telluride detectors for the qualitative analysis of diagnostic $\mathrm{x}$-ray spectra. Phys Med Biol 29, 1117-1131.

[7] Geraldelli, W., Tomal, A., Poletti, M.E., 2013. Characterization of TissueEquivalent Materials Through Measurements of the Linear Attenuation
Application of a CdTe Detector for

Measurements of Mammographic X-ray Spectra 
Santos, J.C. Tomal, A. Costa, P.R.
Coefficient and Scattering Profiles Obtained With Polyenergetic Beams. IEEE Transactions on Nuclear Science 60, 566-571.

[8] Hammerstein, R.G., Miller, D.W., White, D.R., Ellen Masterson, M., Woodard, H.Q., Laughlin, J.S., 1979. Absorbed Radiation Dose in Mammography. Radiology 130, 485-491.

[9] Hernandez, A.M., Boone, J.M., 2014. Tungsten anode spectral model using interpolating cubic splines: Unfiltered $\mathrm{x}$-ray spectra from $20 \mathrm{kV}$ to $640 \mathrm{kV}$. Medical Physics 41.

[10] Johns, P.C., Yaffe, M.J., 1987. X-ray characterisation of normal and neoplastic breast tissues. Physics in Medicine and Biology 32, 675.

[11] Künzel, R., Herdade, S.B., Terini, R.A., Costa, P.R., 2004. X-ray spectroscopy in mammography with a silicon PIN photodiode with application to the measurement of tube voltage. Medical Physics 31, 2996-3003.

[12] Künzel, R., Levenhagen, R.S., Herdade, S.B., Terini, R.A., Costa, P.R., 2008. $\mathrm{X}$-ray spectroscopy applied to radiation shielding calculation in mammography. Medical Physics 35, 3539-3545.

[13] Meyer, P., Buffard, E., Mertz, L., Kennel, C., Constantinesco, A., Siffert, P., 2004. Evaluation of the use of six diagnostic X-ray spectra computer codes. Brit. J. Radiol. 77, 224-230.

[14] Santos, J.C., Mariano, L., Tomal, A., Costa, P.R., 2016. Evaluation of conversion coefficients relating air-kerma to $\mathrm{H}^{*}(10)$ using primary and transmitted $\mathrm{x}$-ray spectra in the diagnostic radiology energy range. Journal of Radiological Protection 36, 117-132.

[15] Santos, J.C., Tomal, A., Furquim, T.A., Fausto, A.M.F., Nogueira, M.S., Costa, P.R., 2017. Technical Note: Direct measurement of clinical mammographic x-ray spectra using a CdTe spectrometer. Medical Physics, n/a-n/a.

[16] Stumbo, S., Bottigli, U., Golosio, B., Oliva, P., Tangaro, S., 2004. Direct analysis of molybdenum target generated X-ray spectra with a portable device. Med. Phys. 31, 2763-2770.

[17] Tomal, A., Cunha, D.M., Poletti, M.E., 2013. Optimal X-ray spectra selection in digital mammography: A semi-analytical study. IEEE Transactions on Nuclear Science 60, 728-734.

[18] Tomal, A., Cunha, D.M., Poletti, M.E., 2014. Comparison of beam quality parameters computed from mammographic x-ray spectra measured with different high-resolution semiconductor detectors. Radiation Physics and Chemistry 95, 217-220.

[19] Tomal, A., Santos, J.C., Costa, P.R., Lopez Gonzales, A.H., Poletti, M.E., 2015. Monte Carlo simulation of the response functions of CdTe detectors to be applied in X-ray spectroscopy. Appl Radiat Isot 100, 32-37.

[20] Tucker, D.M., Barnes, G.T., Wu, X., 1991. Molybdenum target x-ray spectra: A semiempirical model. Med. Phys. 18, 402-407. 